\title{
A Stroke in the Young with Surprising Recovery
}

\author{
Dor Cohen ${ }^{1}$, Gila Shpitalni $^{1,2}$, Yulia Lifshitz $^{1}$, Sapir Anani $^{1}$, Ana Eyal $^{3}$, Gad Segal $^{1,2}$ \\ ${ }^{1}$ Department of Internal Medicine T, Chaim Sheba Medical Center, affiliated to the Sackler Faculty of Medicine, Tel-Aviv University, Tel Aviv, Israel \\ ${ }^{2}$ St. George's, University of London School of Medicine, delivered by Nicosia University at the Chaim Sheba Medical Center, Tel Aviv, Israel \\ ${ }^{3}$ Department of Diagnostic Imaging, Chaim Sheba Medical Center, affiliated to the Sackler Faculty of Medicine, Tel-Aviv University, Tel Aviv, Israel
}

Received: $13 / 03 / 2019$

Accepted: $28 / 04 / 2019$

Published: $24 / 05 / 2019$

How to cite this article: Cohen D, Shpitalini G, Lifshitz Y, Anani S, Segal G. A stroke in the young with surprising recovery. EJCRIM 2019;6: doi:10.12890/2019_000200.

Conflicts of Interests: The Authors declare that there are no competing interests.

This article is licensed under a Commons Attribution Non-Commercial 4.0 License

\section{ABSTRACT}

Introduction: The differential diagnosis of stroke in a comparatively young adult should always include cardiovascular aetiologies as well as central nervous system infection.

Case Presentation: A 56-year-old man, with no significant medical history, presented with headache, nausea and vomiting, and right hemiparesis. Routine stroke investigation was initiated, while CNS infection was also sought. Diagnoses of HIV infection, neurosyphilis, HCV and HBV were established. Targeted therapy resulted in prompt clinical improvement.

Conclusion: This case highlights the importance of considering CNS infection as a cause of neurological deficits in parallel with other investigations in cases of stroke in a comparatively young adult.

\section{LEARNING POINTS}

- The differential diagnosis should be wide for all patients presenting with stroke.

- Neurosyphilis should be included in the differential diagnosis of stroke in the young and middle-aged.

- Newly diagnosed HIV patients should be screened for other, sexually transmitted coinfection.

\section{KEYWORDS}

Stroke in the young, neurosyphilis, AIDS, HIV, syphilis, HCV

\section{CASE DESCRIPTION}

A 56-year-old man with no significant medical history presented to hospital with right hemiparesis which had developed gradually over 2-3 days. He had recently immigrated to Israel from Russia, spoke very little Hebrew and worked as a janitor. After initial workup, as the symptoms had lasted for over 24 hours, the patient was admitted to the internal medicine department with suspected stroke since there were no vacant beds in the neurology department. Vital signs were normal at admission. The patient's general appearance was unkempt; substance abuse, promiscuous sexual activity and exotic travel were denied. His physical examination revealed normal heart sounds without murmurs and bilateral vesicular breathing sounds on both lungs. The abdomen was soft and non-tender without any evidence of organomegaly. There was no evidence of pedal oedema. Skin examination revealed tinea unguium on all digits of the left hand. The patient had prominent facial seborrheic dermatitis. Neurological examination revealed mild seventh cranial nerve palsy on the left with right hemiparesis (strength of $3 / 5$ in the right lower and upper limbs). Further investigation revealed abnormal liver function tests with elevated AST (139 IU/I), ALT (85 IU/I) and GGT (282 IU/I). CT of the brain (without contrast material) was normal. An electrocardiogram showed normal sinus rhythm. A posterioranterior chest $\mathrm{x}$-ray was normal. 
Routine workup and management of stroke in a middle-aged adult were initiated upon admission. The patient's comparatively young age, low socioeconomic status and the fact that he was an immigrant, along with the dermatological findings, suggested CNS infection should be ruled out. Serological testing using the HIV combo test and immunoblot assay, HBV-hepatitis B core antigen (S/CO) and hepatitis B antisurface (HbsAg) antibody (mlU/ml) including hepatitis Be antigen, $\mathrm{HCV}$-hepatitis $\mathrm{C}$ antibody (S/CO) and syphilis-RPR, were all positive (Table 1). The CD4 count was 230 cells $/ \mathrm{mm}^{3}$. The opening pressure of a lumbar puncture was $160 \mathrm{~cm} \mathrm{H}_{2} \mathrm{O}$. The CSF cell count was 66 cells/mm (PMN cells and mononuclear cells), the protein level was elevated $(64 \mathrm{mg} / \mathrm{dl}$ ), and glucose and lactate levels were within the normal range. CSF real-time PCR was positive for JC virus but negative for cryptococcal antigen, Toxoplasma gondii, Toxoplasma gondii IGM and syphilis VDRL. Culture for mycobacteria was negative.

The initial CT scan of the brain mentioned above was normal. However, MRI of the brain showed subacute stroke in the medulla together with general subcortical microangiopathic changes. Several characteristic findings associated with neurosyphilis were also seen. These included general atrophy, mesial temporal atrophy (Fig. 1) as well as an infarct in the midline medulla and left pyramid (Figs. 2 and 3). Further CT angiographic studies ruled out large-vessel vasculitis and other significant vascular anomalies. Trans-thoracic echocardiography showed no evidence of endocarditis.

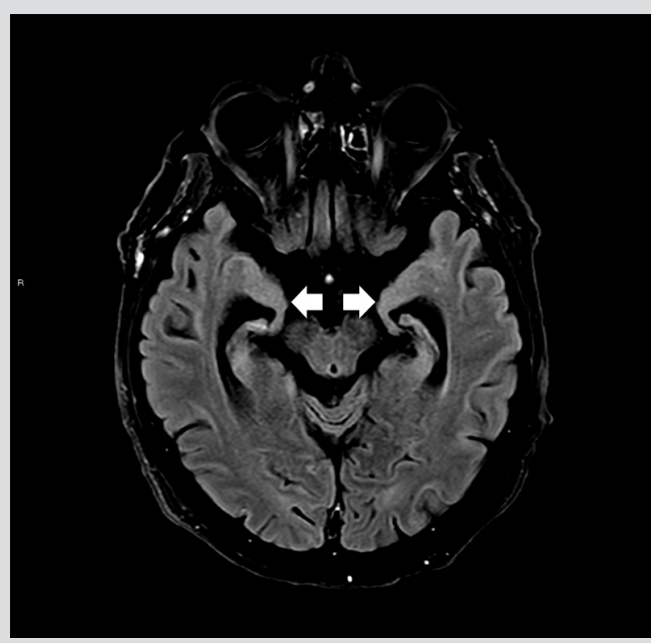

Figure 1. T2 FLAIR weighted image showing symmetrical atrophy (white arrowheads) of the mesial temporal lobes

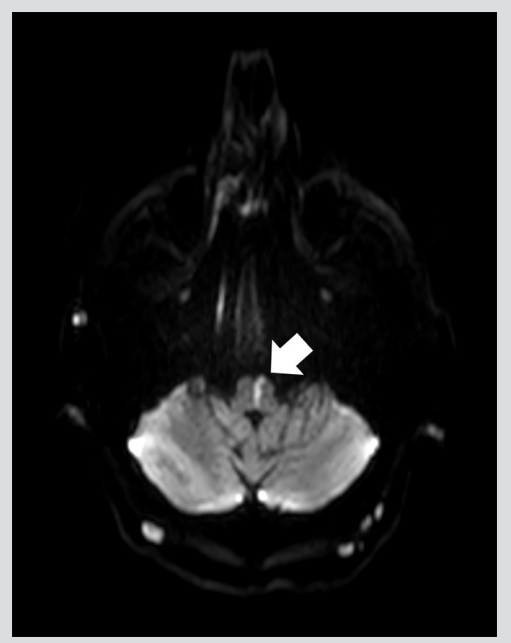

Figure 2. Diffusion weighted MRI image showing an infarct (white arrowhead) in the acute phase midline medulla and left pyramid

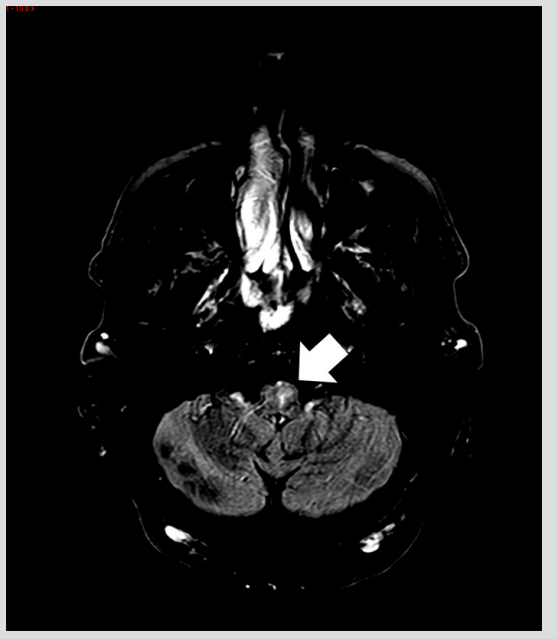

Figure 3. T2 FLAIR MRI image showing an acute infarct (white arrowhead) in the midline medulla and left pyramid

\begin{tabular}{|c|c|c|c|c|c|}
\hline & Patient & Normal Range & & Patient & Normal Range \\
\hline \multicolumn{3}{|l|}{ Cerebrospinal fluid } & \multicolumn{3}{|l|}{ Hepatitis serology } \\
\hline Glucose (mg/dl) & 50 & $50-80$ & Hepatitis B core total (S/CO) & 6.8 & \\
\hline Lactate (mg/dl) & 18.4 & $10-25$ & Anti-HBsAg (m/U/ml) & 205 & \\
\hline Protein (mg/dl) & 64.8 & $22-38$ & Hepatitis Be antibody (S/CO) & 0.02 & \\
\hline WBC (cells $\left./ \mathrm{mm}^{3}\right)$ & 66 & $0-5$ & Hepatitis C antibody (S/CO) & 15.08 & \\
\hline VDRL antigen & Negative & - & HIV immunoblot & Positive & \\
\hline Cryptococcal antigen & Negative & - & Syphilis RPR & 1:32 & \\
\hline \multirow{2}{*}{$\begin{array}{l}\text { Toxoplasma PCR and } \\
\text { toxoplasma IGM }\end{array}$} & Negative & & \multirow{4}{*}{ Syphilis CMIA (S/CO) } & \multirow[t]{4}{*}{21.88} & \\
\hline & & & & & \\
\hline$J C$ virus $R T-P C R$ & Positive & & & & \\
\hline TB culture & Negative & & & & \\
\hline
\end{tabular}

Table 1. Laboratory investigation results

HBsAg, hepatitis B surface antigen; PCR, polymerase chain reaction; RT-PCR, real-time polymerase chain reaction; TB, tuberculosis. 
After a working diagnosis of neurosyphilis was established, the patient was treated with intra-muscular benzathine penicillin $\mathrm{G}$ (for syphilis) and intravenous penicillin G sodium (for neurosyphilis) for 10 days. There was no Jarisch-Herxheimer reaction. Anti-retroviral treatment with a combination of dolutegravir, tenofovir and emtricitabine for HIV was also initiated. The patient received daily physiotherapy. Neurological improvement was noted and after 30 days of hospitalization, the patient was discharged and referred for continued rehabilitation.

\section{DISCUSSION}

The aetiology of stroke in comparatively young adults is diverse and varies according to age and geographical region. Non-cerebrovascular stroke accounts for $20-30 \%$ of cases. A wide variety of different and uncommon causes of stroke in young adults is described in the literature and includes non-atherosclerotic angiopathies, antiphospholipid syndrome, sickle cell disease, inherited syndromes (e.g., mitochondrial encephalopathy, lactic acidosis and stroke-like episodes (MELAS) and Fabry disease), and inflammatory and infectious agents (including HIV, neurosyphilis and Varicella zoster virus), along with several types of vasculitides ${ }^{[1]}$.

Syphilis is a sexually transmitted, chronic systemic infection caused by Treponema pallidum. Prevalence in developing countries can reach $25 \%$ among blood donors. Israel receives immigrants from all over the world, particularly from the former Soviet Union and Africa. The WHO European Health database shows that Eastern European and African countries have a high incidence of syphilis ${ }^{[2]}$.

In newly diagnosed HIV patients who present with focal neurological findings, the differential diagnosis includes toxoplasmosis, progressive multifocal leukoencephalopathy due to JC virus, CNS lymphoma and stroke ${ }^{[3]}$. Neurosyphilis can present at any stage of the disease. The clinical categories of symptomatic neurosyphilis include meningeal, meningovascular and parenchymatous syphilis. Meningovascular disease, through the mechanism of small, medium and large vessel arteritis, can manifest as an infarct indistinguishable from cerebrovascular stroke ${ }^{[4]}$. The diagnosis of neurosyphilis in HIV patients can be difficult for several reasons: (a) CSF serology for VDRL may only be positive in approximately one third of cases; (b) HIV infection itself can cause CSF pleocytosis and an increased protein level; and (c) HIV-associated vasculopathy can contribute to inflammation of the cerebral blood vessels through different mechanisms such as vasculitis, accelerated atherosclerosis and aneurysm formation ${ }^{[5,6]}$. Brain MRI findings may demonstrate characteristic but non-specific findings of neurosyphilis, including general atrophy and mesial temporal atrophy ${ }^{[7]}$, as well as infarct in the midline medulla and left pyramid (as described in our patient).

This case highlights the importance of considering CNS infections as a cause of neurological deficits in conjunction with other investigations in comparatively young patients presenting with stroke. Thrombolysis was not administered to our patient due to the delay between clinical presentation and treatment initiation. The gradual onset of symptoms should always suggest infection as the cause of a stroke in progress. In their retrospective review of 53 patients with syphilitic vasculitis affecting the central nervous system, Ahbeddou et al. described a prodromal phase preceding signs of stroke ${ }^{[8]}$. We do not know the effect thrombolysis would have had in our patient, since there is no relevant literature. Our case also highlights the complexity of the management of newly diagnosed HIV patients with syphilis coinfection. Even with a non-reactive CSF VDRL test, the combination of newly diagnosed HIV infection together with positive syphilis serum serology, new onset of focal neurological findings and pathological CNS findings is sufficient to prompt treatment initiation.

\section{REFERENCES}

1. Smajlović D. Strokes in young adults: epidemiology and prevention. Vasc Health Risk Manag 2015;11:157-164.

2. Eilam A, Grin D, Avishay S, Gilad R. Neurosyphilis presenting with a stroke-like syndrome. J Neuroinfect Dis 2017;8:254.

3. Jameson JL, Kasper DL, Longo DL, Fauci AS, Hauser SL, Loscalzo J, editors. Harrison's principles of internal medicine, 20th ed. New York: McGraw-Hill Education; 2018, p. 1145-1446.

4. Jan K, Min RHH, Yen TS, Singh SR. Ischemic stroke in an HIV positive patient: an initial presentation of neurosyphilis. Case Rep Neurol Med 2018;2410154.

5. Katelaris AL. Medial medullary stroke due to neurosyphilis in a newly diagnosed HIV-positive man. Intern Med J 2018;48:992-995.

6. Benjamin LA, Bryer A, Emsley HCA, Khoo S, Solomon T, Connor MD. HIV infection and stroke: current perspectives and future directions. Lancet Neurol 2012;11(10):878-890.

7. Nagappa M, Sinha S, Taly AB, Rao SL, Nagarathna S, Bindu PS, et al. Neurosyphilis: MRI features and their phenotypic correlation in a cohort of 35 patients from a tertiary care university hospital. Neuroradiology 2013;55(4):379-388.

8. Ahbeddou N, El Alaoui Taoussi K, Ibrahimi A, Ait Ben Haddou EH, Regragui W, Benomar A, et al. Stroke and syphilis: a retrospective study of 53 patients. Rev Neurol (Paris) 2018;174(5):313-318. 